\title{
Conventional Taxonomy of Four Tilapia Species in South-East Coast of India
}

\author{
Viki R.V., Sathya A.C.R.* and Muthu M.S. \\ Centre of Advanced Study in Marine Biology, Annamalai University, India \\ *acrathish@gmail.com
}

\begin{abstract}
Tilapia belongs to the family Cichlidae. Tilapia is a generic term used to designate a group of commercially important food fishes belonging to the family Cichlidae. Tilapia species have been re-classified into three separate genera viz., Tilapia, Sarotherodon and Oreochromis, based mainly on their reproduction and feeding habits and biogeography. Tilapias are very sturdy in nature. The family Cichlidaeis is one of the most species-rich families of vertebrates. More than 3,000 species of cichlid fishes are distributed from Central and South America, through Africa and Madagascar to Southern India. Classification of species is fundamental for carrying out researches in biodiversity, ecology, evolutionary biology and conservation biology. With this as the background in the present study, four species of fishes namely $O$. mossambicus and O.niloticus, Tilapia rendalli and T.zilliiare were sequenced for their $647 \mathrm{bp}$, $645 \mathrm{bp}, 642$ and 481 region of cytochrome oxidase sub-unit I (COI) gene to test their efficacy in identifying the species and also to demonstrate their intra-species variations within the barcode region. The sequences were analyzed for their species identification, using the Barcode of Life Database (BOLD's) identification engine. The COI sequences of O. mossambicus, O.niloticus, Tilapia rendalli and T.zillii from different geographical regions were extracted from NCBI for analyzing the intra-species variation. While comparing the sequences in the NCBI database with the present sequences, significant alignments with maximum similarity ranging from $99 \%$ to $100 \%$ were noticed. Four sequences are aligned using ClustalW. A phylogenetic tree was constructed with Bootstrap test. The optimal tree with the sum of branch length showed 0.000976 for $O$. mossambicus, 0.001556 for $O$. niloticus and 0.000000 for $T$. rendalli and T.zillii. In O. mossambicus, the maximum GC content was $46.9 \%$ whereas the GC content in all other closely related species was found to be $46 \%$ on an average. In O. niloticus, the maximum GC content of $47.9 \%$ was noticed and the closely related sequences exhibited $48 \%$ average in GC content (T.zillii 50.2\% and T.rendalli 46.6\%). The analyses revealed the molar concentration of bar-coded genes.
\end{abstract}

The evolutionary relationship was inferred with the closely related sequences obtained from the NCBI database. Sequence divergence between individuals of the same species ranged from 0 to 0.057 . Both phylography and phylogeographic signals were evident from the phylogram constructed with $O$. mossambicus and O.niloticus as the same group. Even though the present results confirm that the COI could be a potential barcode gene for species level identification, further research is needed for describing the divergence of sequences in a broader sense. Analysis of data undertaken with conventional tools like PCR amplification and gene sequencing methods clearly revealed the healthy nature of environment along the southeast coast of India.

Keywords: Intra species, Variations, Barcode, Cytochrome oxidase, Phylogenetic

Proceedings of the International Forestry and Environment Symposium 2016, Department of Forestry and Environmental Science, University of Sri Jayewardenepura, Sri Lanka. 